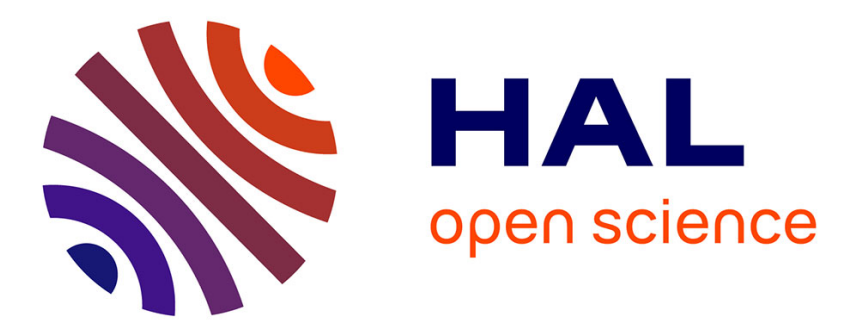

\title{
Magnetic manipulation with several mobile coils towards gastrointestinal capsular endoscopy.
}

\author{
Baptiste Veron, Joël Abadie, Arnaud Hubert, Nicolas Andreff
}

\section{To cite this version:}

Baptiste Veron, Joël Abadie, Arnaud Hubert, Nicolas Andreff. Magnetic manipulation with several mobile coils towards gastrointestinal capsular endoscopy.. Fernando Viadero, Marco Ceccarelli. New Trends in Mechanism and Machine Science. Theory and Applications in Engineering., SPRINGER, pp.681-690, 2012, Mechanisms and Machine Science 7, 978-94-007-4901-6. 10.1007/978-94-007-49023. hal-00772408

\section{HAL Id: hal-00772408 https://hal.science/hal-00772408}

Submitted on 10 Jan 2013

HAL is a multi-disciplinary open access archive for the deposit and dissemination of scientific research documents, whether they are published or not. The documents may come from teaching and research institutions in France or abroad, or from public or private research centers.
L'archive ouverte pluridisciplinaire HAL, est destinée au dépôt et à la diffusion de documents scientifiques de niveau recherche, publiés ou non, émanant des établissements d'enseignement et de recherche français ou étrangers, des laboratoires publics ou privés. 


\title{
Magnetic manipulation with several mobile coils towards gastrointestinal capsular endoscopy.
}

\author{
Baptiste Véron, Joël Abadie, Arnaud Hubert and Nicolas Andreff
}

FEMTO-ST / AS2M, France, e-mail: baptiste.veron@femto-st.fr

\begin{abstract}
Traditional techniques of endoscopy based on flexible endoscopes are fairly reliable but poorly tolerated by patients and do not give access to the small bowel. It has been demonstrated that magnetic fields are usable for manipulating an untethered magnet, either using fixed coils or mobile permanent magnets. We introduce a novel approach for magnetic manipulation and present the preliminary results obtained by simulating a planar manipulation system including multiple mobile coils.
\end{abstract}

Key words: magnetic manipulation, capsule endoscope, variable magnetic field

\section{Introduction}

\subsection{Exploration of the gastrointestinal tract}

Traditional techniques for exploring the gastrointestinal tract are based on endoscopy. These techniques, based on flexible endoscopes, are fairly reliable and provide high resolution images that enable accurate diagnosis. However these procedures present several drawbacks. First, the physician needs much practice to acquire the necessary dexterity to manipulate the endoscope. Second, movements done by the endoscope inside the body are frequently painful and traumatic. Whole body anesthesia may be a solution to these pain problems but it is not always possible considering the patient's age and history. And last, these procedures do not allow observation of the whole small bowel.

In order to access small bowel and improve gastrointestinal diagnosis techniques, Given Imaging developed, in 2000, the M2A (mouth to anus) capsule endoscopy system [18]. Approved by FDA in August of 2001, under the Pillcam name, it is a $11 \times 26 \mathrm{~mm}$ disposable capsule embedding a battery, a CCD camera, LEDs and a $\mathrm{RF}$ transmitter. Patients swallow the capsule which will move in the gastrointestinal tract thanks to peristalsis (natural movements of the intestine) while taking 2 pictures per second during the 8 hours of battery life. 
This technology has its greatest utility in the evaluation of obscure gastrointestinal bleeding (bleeding of unknown origin that persists or recurs after a negative initial or primary colonoscopy and/or upper endoscopy) [19]. Indeed, no cause for the bleeding is evident even after both colonoscopy and enteroscopy in approximately $5 \%$ of all patients with gastrointestinal bleeding [10]; and lesions in the small bowel have been found for $27 \%$ of patients with obscure gastrointestinal bleeding [3].

Although capsule endoscopy has permitted great progress, some drawbacks remain. The inability to control the capsule's movements combined with the poor frame rate ( 2 images per second) cause a continuity problem: if the speed of the capsule is too high, there will not be enough images to have a continuous view of the intestine and one might miss something important. Another problem is the location of the capsule. Today, one considers that the location is function of the transit time but because of the irregular speed, this is irrelevant.

Considering these drawbacks, investigation has been done to improve capsule endoscopy by replacing passive locomotion (peristaltis) by an active control of the motion.

\subsection{Capsule active locomotion}

One way of controlling the capsule motion is to put actuators in the capsule. That was studied in the EU VECTOR project with legged capsules [2]. These legs may be made of bioinspired material to achieve a better contact on the small bowel internal wall [7]. Another idea for the active locomotion is based on inchworm-like motion. A shape memory alloy spring moves a gripping part and pushes the capsule [8].

Instead of having embedded actuators, an external actuation method is also possible. Magnetic manipulation is one of the most popular way for capsule motion control because it presents many advantages. Indeed magnetic fields are not toxic to the human body [14] and it permits remote effects without any contact (potentially traumatic) with the intestinal wall. The main idea of magnetic manipulation is to create efforts onto a magnetic part located in an external magnetic field, but the issue is to produce the proper external field that will induce the desired magnetic forces. For that, two approaches can be found in the literature.

The first method is to use static coils and to control their currents to adjust the magnetic field (as in opthalmology) [17, 9]. If a Helmholtz configuration is used, then the workspace is between the two coils and one obtains uniform magnetic fields $[16,15]$. This is also done in magnetic resonance imaging (MRI) systems to move a magnetic micro-object in blood vessels [6, 12]. If the object one wants to manipulate is not made of magnetic material, it is possible to use an indirect way to move it with magneto-sensitives bacteria that move along the field lines [13] created by the MRI system. The second method to create the appropriate external magnetic field is to use permanent magnets and to move them around the patient with a robotic system to steer the manipulated object $[5,1]$. Thus, two separate techniques are used to create the external magnetic field: static coils or mobile permanent magnets. The 
principle is to adjust either the magnetic field strength by a current control in the coils, or changing the local form of the field by modifying the relative position and orientation between the mobile magnet and the magnetic part.

This paper proposes a hybrid approach by using multiple mobile coils in order to control the motion of a magnetic capsule by both servoing the currents and moving the magnetic sources. This opens the ability to create a redundant system because one will be able to modify the magnetic field both mechanically (by moving the coils) and electrically (by adjusting the current in each coil). The aim of this redundancy is to obtain more manipulability and dexterity. In order to verify this, a study of a planar manipulation system is considered.

This paper is organized as follows. Section 2 presents the theoretical background for a new approach of magnetic manipulation, while Section 3 presents the results obtained by application of the theory on a planar manipulation system simulated with Matlab. Conclusion and perspectives are given in Section 4.

\section{A novel approach, study of a first case}

To manipulate the capsule, an original architecture is proposed. As shown on Fig. 1, the system consists of a small permanent magnet fixed to the capsule we want to manipulate, and three coils that can turn around a vertical axis. This architecture is therefore similar to a 3RPR parallel manipulator with magnetic virtual actuators.

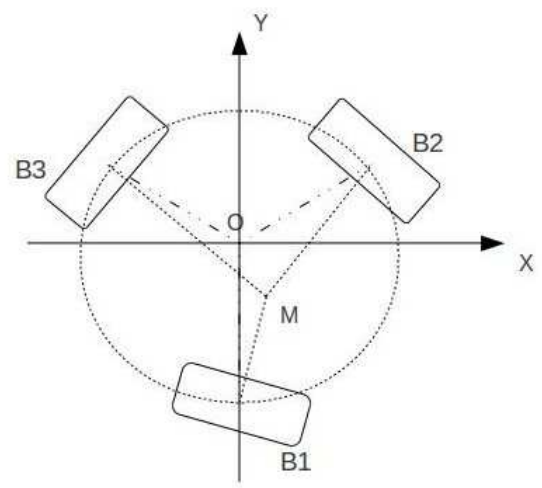

Fig. 1 Architecture of the magnetic manipulation system. B1, B2 and B3 are the three moving coils and $\mathrm{M}$ is the position of the magnet.

Two parameters (orientation and current) per coil are controlled, which makes six parameters for three degrees of freedom. The redundancy is managed by pointing the coil's axis in the direction of the permanent magnet. The 3RPR system can thus be taken as a known reference. 
The magnet motion is driven by the efforts applied on it, as expressed in (1). This system is solved, knowing the mass $m$, the inertia $\left[I_{G}\right]$ of the magnet, and the magnetic efforts created by each coil, for a desired acceleration a and angular acceleration $\dot{\omega}$ (friction is neglected).

$$
\left\{\begin{aligned}
\sum_{i=1}^{3} \mathbf{F}_{m, i} & =m \mathbf{a} \\
\sum_{i=1}^{3} \mathbf{C}_{m, i} & =\left[I_{G}\right] \dot{\omega}
\end{aligned}\right.
$$

The magnetic efforts applied on a permanent magnet located in $P$ with a magnetization $\mathbf{M}$ and a volume $V$ in an external magnetic field $\mathbf{B}_{i}(P)$, are given by [4]:

$$
\left\{\begin{array}{l}
\mathbf{F}_{m, i}=V \cdot \operatorname{grad}\left(\mathbf{B}_{i}(P) \cdot \mathbf{M}\right) \\
\mathbf{C}_{m, i}=V \cdot \mathbf{M} \wedge \mathbf{B}_{i}(P)
\end{array}\right.
$$

Furthermore, the magnetic field created at position $P$ by a circular current loop in free space as shown on Fig. 2 is computed in spherical coordinates as

$$
\mathbf{B}_{i}(P)=\left(\begin{array}{l}
B_{r}=\frac{\mu_{0}}{2 \pi}\left(I_{i} \cdot \pi a^{2}\right) \frac{\cos \theta}{r^{3}} \\
B_{\theta}=\frac{\mu_{0}}{2 \pi}\left(I_{i} \cdot \pi a^{2}\right) \frac{\sin \theta}{r^{3}} \\
B_{\phi}=0
\end{array}\right)
$$

where $\mu_{0}$ is the permeability of free space, $a$ the current loop radius, $r$ the distance between $O$ and $P$, and $\theta$ the angle $(\widehat{\mathbf{Z , O P}})$.

For a $\mathrm{N}$ turns coil, all loops are considered at the same point so the previous formula is multiplied by $\mathrm{N}$ to obtain the magnetic field. Then superposition principle is applied to find the magnetic field created by all three coils.

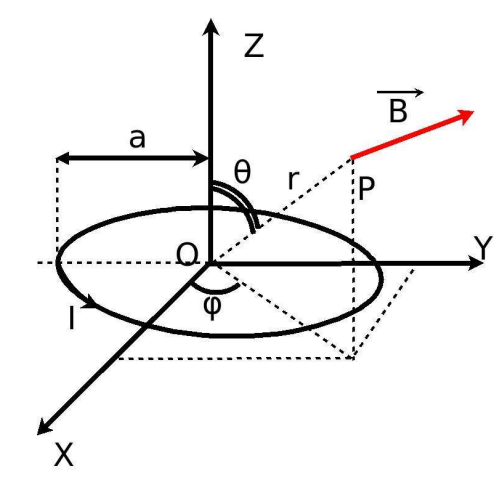

Fig. 2 Magnetic field created by a circular current loop.

Then, given the spatial configuration of the coils, it is possible to find the current in each coil that will allow to move the magnet in the desired direction by applying the proper magnetic field. 
To manage the magnet position $\mathrm{X}$, the magnetic field created by the coils has to be controlled. Therefore the current $\mathbf{I}=\left(I_{1}, I_{2}, I_{3}\right)$ in each coil must be regulated. This is done by using a feedback contol (proportional, derivative, plus feed-forward) which provides the desired acceleration of the moving objects, and solving for the inverse dynamic model of the system (1)-(2). This control scheme is illustrated on Fig. 3.

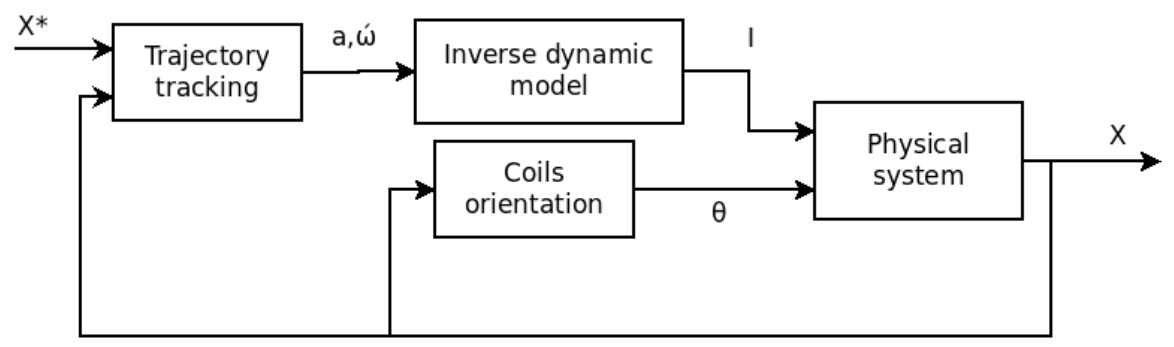

Fig. 3 Control strategy

\section{Results}

\subsection{Simulation with Matlab}

A simulation using Matlab and Simulink was developed to validate the model and the proposed control strategy. This simulation is splited in two parts. One part is a physical simulator for the system [4], and the other part is dedicated to the control of the system.

In the simulation, we assume that the desired trajectory is slow enough and the motion of the coils fast enough to consider the coil control as instantaneous (quasistatic) so that we can focus on the magnetic control only.

Figure 4 presents preliminary results from the simulation. We managed to make the magnet follow several trajectories (Fig. 4(a), 4(b) and 4(c)) which shows that the singularities on this system are not the same as the ones found on a 3RPR parallel manipulation system.

Although these results prove that the chosen control strategy allows the magnetic manipulation, Fig. 4(d) shows a case where control on the magnet orientation is lost. An extended study has to be done to explain this loss of control, but we suspect that it may be caused by a singularity or a numerical problem (ill-conditioned matrix), if not a bug in the simulation. 


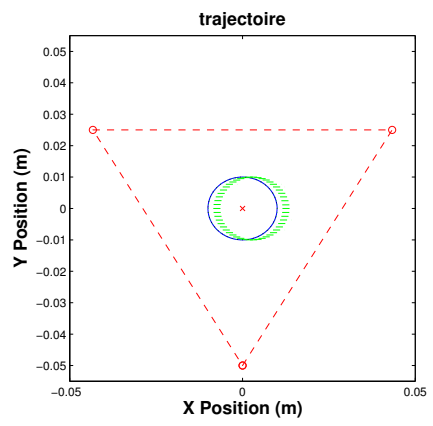

(a) Circle of a $0.01 \mathrm{~m}$ radius.

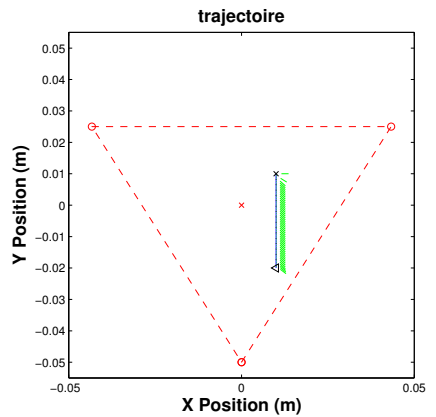

(c) Vertical straignt line with a different orientation.

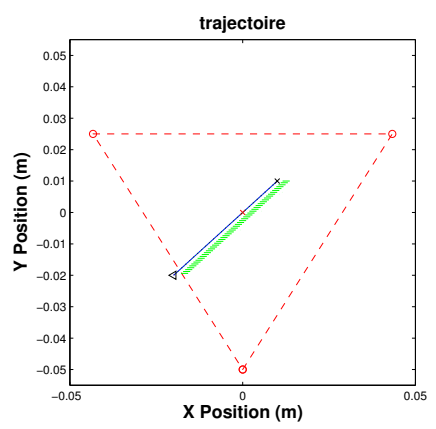

(b) Straight ligne passing through the center.

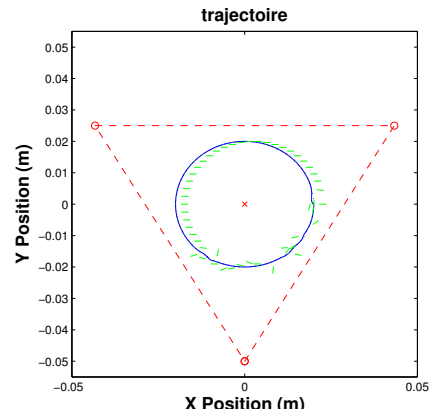

(d) Loss of the motion control on a circle with a $0.02 \mathrm{~m}$ radius.

Fig. 4 Simulation results for several trajectories. In red is the triangle supporting the coils, in blue the magnet trajectory and in green its orientation vector.

\subsection{Prototype}

To confirm the simulation results, a prototype is being developed based on the architecture used in the simulation. This prototype is a modular system in order to be able to test several geometries for the coils position and the dimension of the workspace, as what have been done in the simulation.

The coils and their rotating system are mounted on adjustable platforms (Fig. 5(a)) which are fixed on a static frame. The magnet position is measured with a camera fixed above the workspace as shown on Fig. 5(b).

The software architecture will be based on the simulation (which will be translated to $\mathrm{C}++$ ) and the ViSP library [11] (a C++ cross-platform solution, developed by the INRIA Lagadic team, for prototyping and developing applications in visual tracking and visual servoing). 


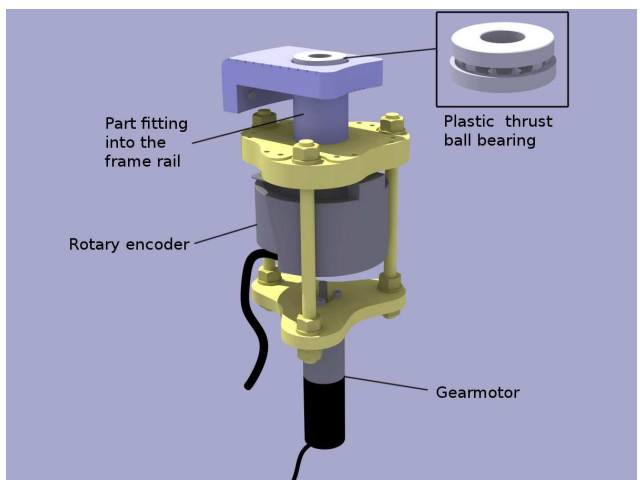

(a)

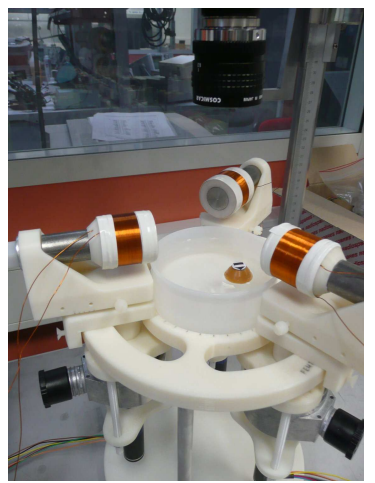

(b)

Fig. 5 (a): CAD model of one platform. (b): Picture of the prototype.

\section{Conclusion and perspectives}

A new paradigm was proposed: the use of mechanical and electromagnetic redundancy for improving the dexterity and manipulability of magnetic manipulation. Feasibility have been shown by simulating a planar manipulation system. More, the simulation results were used to design a prototype. The next steps are to complete the prototype and to study the various control strategies.

In parallel to this work, a study on redundancy management is led. It has been shown that the strategy chosen to manage the coils orientation presents singularities. Therefore, other strategies will be compared to this one to improve the system control.

Moreover, even if the use of a ferromagnetic core in the prototype's coils permits to increase the magnetic field, it makes the basic field model (3) not valid any more. A more realistic model to compute the magnetic field has then to be developed.

This prototype is a first step in magnetic manipulation experiments towards capsular endoscopy. It will help us to have a better understanding of what is happening and how to control it. Even if this prototype is a 2D manipulation system, the proposed model should work in 3D. We expect then to build a 3D demonstrator in order to manipulate capsules through the human body in a rather short future.

Acknowledgements The authors would like to thank Valentin Lehoussel and David Guibert for their help in the prototype design and assembly.

\section{References}

1. Stereotaxis as stargen eu ltd. niobe 2, magnetic navigation system. Stereotaxis niobe system website: http://www.stereotaxis.com/niobe.html. 
2. O. Alonso, L. Freixas, J. Canals, E. Susilo, and A. Diguez. Control electronics integration toward endoscopic capsule robot performing legged locomotion and illumination. In IEEE/RSJ International Conference on Intelligent Robots and Systems, Taipei, Taiwan, October 18-22 2010.

3. M. Appleyard, Z. Fireman, A. Glukhovsky, H. Jacob, R. Shreiver, S. Kadirkamanathan, A. Lavy, S. Lewkowicz, E. Scapa, R. Shofti, P. Swain, and A. Zaretsky. A randomized trial comparing wireless capsule endoscopy with push enteroscopy for the detection of small-bowel lesions. Gastroenterology, 119(6):1431-8, 2000.

4. A. Cherry. Étude, conception et optimisation d'une plateforme de mesure de micro et nano force par flottaison magnétique. PhD thesis, Université de Franche-Comté, 2009.

5. G. Ciuti, P. Valdastri, A. Menciassi, and P. Dario. Robotic magnetic steering and locomotion of capsule endoscope for diagnostic and surgical endolumial procedures. Robotica, 28:199-207, 2010.

6. O. Felfoul, E. Aboussouan, A. Chanu, and S. Martel. Real-time positioning and tracking technique for endovascular untethered microrobots propelled by mri gradients. In IEEE International Conference on Robotics and Automation, 2009.

7. P. Glass, M. Sitti, and R. Appasamy. A new biomimetic adhesive for therapeutic capsule endoscope applications in the gastrointestinal track. Gastrointestinal Endoscopy, 65(5):AB91, 2007.

8. B. Kim, S. Lee, J. H. Park, and J.-O. Park. Design and fabrication of a locomotive mechanism for capsule-type endoscopes using shape memory alloys (smas). In IEEE/ASME Transaction on Mechatronics, volume 10(1), pages 77-86, 2005.

9. M. P. Kummer, J. J. Abbott, B. E. Kratochvil, R. Borer, A. Sengul, and B. J. Nelson. Octomag: An electromagnetic system for 5-dof wireless micromanipulation. In 2010 IEEE International Conference on Robotics and Automation, pages 1610-1616, 2010.

10. S. Lahoti. The small bowel as a source of gastrointestinal blood loss. Curr Gastroenterol Rep, 1(5):424-30, 1999.

11. E. Marchand, F. Spindler, and F. Chaumette. Visp for visual servoing: a generic software platform with a wide class of robot control skills. IEEE Robotics \& Automation Magazine, 12 (4):40-52, December 2005.

12. S. Martel, J.-B. Mathieu, O. Felfoul, A. Chanu, E. Aboussouan, S. Tamaz, P. Pouponneau, L. Yahia, G. Beaudoin, G. Soulez, and M. Mankiewicz. Automatic navigation of an untethered device in the artery of a living animal using a conventional clinical magnetic resonance imaging system. In Applied physics letters, 2007.

13. S. Martel, A. Walder, M. Mohammadi, Z. Lu, and O. Felfoul. Towards swarms of communication-enabled and intelligent sensotaxis-based bacterial microrobots capable of collective tasks in an aqueous medium. In 2009 IEEE International Conference on Robotics and Automation, 2009.

14. J. F. Schenck, C. L. Dumoulin, R. W. Redington, H. Y. Kressel, R. T. Elliott, and I. L. McDougall. Human exposure to 4.0 tesla magnetic fields in a whole-body scanner. Medical Physics, The International Journal of Medical Physics Research and Practice, 19(4):10891099, 1992.

15. X. Wang. Study on Magnetic Localization and Actuation of Active Capsule Endoscope. PhD thesis, The Chinese University of Hong Kong, August 2006.

16. X. Wang and M. Q.-H. Meng. A magnetic stereo-actuation mechanism for active capsule endoscope. In Proceedings of the 29th Annual International Conference of the IEEE EMBS, pages 2811-2814, 2007.

17. K. B. Yessin, K. Vollmers, and B. J. Nelson. Modeling and control of untethered biomicrorobots in a fluidic environment using electromagnetic fields. The International of Robotics Research, 25:527, 2006.

18. M. Yu. M2a (tm) capsule endoscopy: A breakthrough diagnostic tool for small intestine imaging. Gastroenterology Nursing, 25(1):24-27, 2002.

19. G. R. Zuckerman, C. Prakash, M. P. Askin, and B. S. Lewis. Aga technical review on the evaluation and management of occult and obscure gastrointestinal bleeding. Gastroenterology, $1: 118,2000$. 